\title{
COMUNICARE LA SCIENZA MEDICA NEL SETTECENTO EUROPEO: UNO SGUARDO ALLE PROSPETTIVE DI RICERCA
}

\author{
ELENA AGAZZI (*), GIOVANNI IAMARTINO (**), \\ PAOLO MAZZARELLO $(* * *)$
}

Gli interventi sulla storia settecentesca della scienza medica che hanno dato vita alla giornata di studio presso l'Istituto Lombardo Accademia di Scienze e Lettere, e che ora sono raccolti in questo volume, non costituiscono solo degli interessanti studi di caso, ma rivelano tutta la ricchezza e la complessità del tema affrontato. Infatti e innanzitutto, il Settecento medico si prolunga lungo un'estensione temporale culturalmente polimorfa caratterizzata da contrapposizioni marcate di concezioni sviluppate su radici antiche, ma anche di sintesi degli opposti nella prospettiva di un nuovo inizio. Secondariamente, è evidente la prospettiva europea della riflessione medico-scientifica settecentesca, che travalica i confini nazionali per un dialettico scambio di idee, suggestioni, e testi. In terzo luogo, si nota un progressivo movimento peraltro, già avviato nel secolo precedente ma con tempi e ritmi differenti nelle diverse aree culturali - dal latino alle lingue nazionali. Infine, al progressivo approfondirsi delle conoscenze mediche corrisponde una più ampia apertura verso la loro divulgazione, poiché tali saperi non devono rimanere chiusi all'interno della stretta cerchia degli specialisti ma, in buona misura, interessano e devono interessare un po'

(*) Istituto Lombardo Accademia di Scienze e Lettere; Università degli Studi di Bergamo, Italia. E-mail: elena.agazzi@unibg.it

${ }_{(* *)}$ Istituto Lombardo Accademia di Scienze e Lettere; Università degli Studi di Milano, Italia. E-mail: giovanni.iamartino@unimi.it

${ }^{(* * *)}$ Istituto Lombardo Accademia di Scienze e Lettere; Università degli Studi di Pavia, Italia. E-mail: paolo.mazzarello@unipv.it 
tutte le persone istruite; ne consegue che il discorso medico-scientifico 'contamina' produttivamente altre forme della riflessione e comunicazione umana, prime fra tutte la filosofia e la letteratura.

Non a caso la giornata di studio presso l'Istituto Lombardo Accademia di Scienze e Lettere ha visto la proficua interazione di specialisti di diverse discipline: non solo storici e storici della medicina, ma anche cultori di storia delle letterature, delle culture e delle lingue - che hanno declinato in una pluralità di temi, di approcci e di contesti socio-culturali diversi il nucleo scientifico dell'incontro, come risulterà chiaro dai diversi contributi qui presentati.

I primi due si focalizzano sull'area culturale di lingua tedesca, mostrando in modi diversi quando sia complesso delimitare il campo d'azione della scienza medica settecentesca. Infatti, il contributo di Jürgen Barkhoff sul mesmerismo chiarisce come questa pratica terapeutica, che si deve al medico tedesco Anton Mesmer (1734-1815), si colloca al confine tra la scienza popolare, l'esoterismo e gli studi sull'elettricità nervosa. Mesmer analizzava l'eccitabilità dei corpi alla luce di una teoria dei fluidi che seminò molte perplessità tra i fautori del razionalismo illuminato. Barkhoff indaga, su tre diversi livelli, il fenomeno del mesmerismo: quello tra magnetizzatore e paziente, quello tra corpo e mente, e quello tra esseri umani e dimensione cosmica, in cui, tramite una terapia che intendeva ristabilire la corretta circolazione del fluido magnetico, l'individuo poteva ritrovare la propria armonia. Il mesmerismo si scontrò con molte resistenze e preoccupazioni: si espose al sospetto di cialtroneria, ma fu investito anche da accuse di cospirazione antimonarchica. Ciò accadde perché la figura del magnetizzatore sembrava poter assumere il pieno controllo sugli individui, alienandoli dalla realtà. In questo modo, risultava minata la fiducia nella superiorità della ragione sull'inconscio. Barkhoff sottolinea tuttavia che, da un punto di vista comunicativo, il mesmerismo poté contare anche su un'ampia partecipazione, concedendo spazio a una spettacolarizzazione delle pratiche terapeutiche nelle cosiddette 'stanze della crisi', in cui si organizzavano sedute curative di gruppo. Nelle sedute si adottava l'uso di strumenti di grande impatto emotivo, tra i quali la Glassarmonica (nota è la scena nella Lucia di Lammermoor in cui questo strumento formato da bicchieri sottolinea con i suoi toni mesti la pazzia della protagonista). Si può dunque affermare che il mesmerismo, collocandosi nel quadro della cultura scientifica tra XVIII e XIX secolo, contribuì a segnare il mutato clima culturale e intellettuale tra Illuminismo e Romanticismo. 
Quest'ultima corrente di pensiero, infatti, si appropriò degli stati di alienazione dell'anima e li tradusse produttivamente in forme di liberazione dalle costrizioni del corpo e di raggiungimento di una maggiore consapevolezza di sé. Nella parte conclusiva del suo contributo, Barkhoff asserisce che la letteratura è riuscita a trarre dal mesmerismo maggiori vantaggi rispetto alla scienza, perché ha insistito sui lati oscuri della complessione umana, contribuendo a definire la dimensione del 'perturbante', a tratti demoniaco, nelle opere di E.T.A. Hoffmann, di Jean Paul, di von Arnim e di molti altri scrittori. Il grande quesito su 'dove risieda' e 'dove vada' l'anima negli stati alterati della coscienza, ivi incluso il sogno, occupò a lungo il dibattito europeo tra il XVIII e il XIX secolo.

L'anima umana, che si manifesta nelle passioni attraverso il volto, fu un cardine degli studi medico-filosofici che fiorirono a Halle a partire dagli anni '40 del Settecento. Federica La Manna li indica come antesignani di una cultura medico-antropologica che, per convenzione, si riconduce in Germania al successivo trattato di Ernst Platner, Anthropologie für Aerzte und Weltweise (1772). Stimolata dall'intuizione di uno scambio sinergico di corpo ed anima da parte di Georg Ernst Stahl (1659-1734), la medicina dei medici-filosofi di Halle, che traevano parte dei propri insegnamenti dall'estetica di Alexander Gottlieb Baumgarten e di Georg Friedrich Meier, si concentrò sui meccanismi legati alle passioni umane e produsse studi il cui fulcro era rappresentato da una Seelenlebre ('dottrina dell'anima') posta in relazione alla fisiologia dell'uomo. I medici di Halle - tra i quali spiccano le personalità di Johann Gottlob Krüger, Johann August Unzer, Ernst Anton Nicolai e Johann Christian Bolten - puntarono sull'osservazione dei sintomi del disagio e sull'esperienza diretta del rapporto con il paziente. Non soltanto la qualità degli studi accademici espressi dalla cerchia di Halle, ma anche le doti divulgative di alcuni dei suoi protagonisti, che in riviste come Der Arzt di Unzer seppero coniugare diffusione del sapere scientifico ed esigenze del pubblico, contribuirono a istruire e ad affascinare i lettori; il racconto della malattia era esposto con uno stile narrativo così avvincente, che colpì persino i grandi letterati del periodo classico-romantico come Goethe e Jean Paul. Lo studio delle emozioni umane divenne anche un importante fattore di analisi interdisciplinari, come mostra il frequente ricorso al disegno e alla pittura nella didattica medica del tempo. Anche La Manna registra non poche avversità nei confronti della diffusione di queste teorie, soprattutto da parte 
dei difensori della fisiognomica, tra i quali spicca il teologo svizzero Johann Caspar Lavater, che tra 1775 e il 1778 pubblicò i noti Physiognomische Fragmente, zur Beförderung der Menschenkenntnis und Menschenliebe (Frammenti fisiognomici per la promozione della conoscenza e dell'amore negli esseri umani). Il suo scopo era suggerire un percorso mistico e religioso per interpretare l'interiorità umana partendo dai tratti fissi del volto. Anche in questo caso, la letteratura tedesca captò l'esperienza medica e la tradusse in un registro originale, come mostra la novella di Schiller, Verbrecher aus verlorener Ebre (Delinquente per onore perduto), del 1792.

La natura complessa, mutevole e in qualche misura sfuggente, della trattatistica medico-scientifica settecentesca viene affrontata nel saggio di Irma Taavitsainen, che analizza una serie di scritti medici in lingua inglese per determinarne $\mathrm{i}$ tratti di continuità e di mutamento rispetto al passato. Da anni coordinatrice di un gruppo di ricerca dell'Università di Helsinki dedicato ai cosiddetti scientific thought-styles, l'approccio di Taavitsainen combina la pragmatica storica con le digital humanities avvalendosi di un corpus informatizzato di testi medici del Settecento inglese. L'attenzione non si focalizza, dunque, su specifici autori o trattati ma, sempre sul fondamento di una documentazione testuale, vengono indicate e commentate le principali linee di tendenza in un contesto, quello britannico, che condivide con l'Europa continentale la presenza di metodi e approcci medici in competizione. Tuttavia, esso è caratterizzato da una più ampia e diversificata gamma di testi dedicati alla cura del corpo e delle malattie, dalle opere in latino di studiosi e medici di formazione universitaria ai testi in inglese definiti da diversi livelli di divulgazione. Naturale fondamento della riflessione medica inglese nel Settecento è la creazione, nel secolo precedente, della Royal Society e l'avvio della pubblicazione delle Philosophical Transactions, che mettono al centro l'importanza dell'evidenza empirica e promuovono la pratica di una sperimentazione scientifica replicabile e oggettiva. La documentazione presentata da Taavitsainen riflette anche le migliorie tecnologiche del periodo (in particolare l'uso del microscopio) che si accompagnano a una crescente professionalizzazione della pratica e della riflessione medica. Allo stesso tempo, la documentazione testuale evidenzia quanto il Secolo dei Lumi sta abbandonando: in particolare, il richiamo alle 'autorità' del passato e alla teoria degli umori nei testi scientifici, e i riferimenti alla religione, all'astrologia e alle pratiche magiche in contesto medico. Il saggio si conclude con 
un invito ad approfondire la ricerca sulla divulgazione del pensiero medico-scientifico nei giornali del tempo e nei testi indirizzati ai nonspecialisti e alle classi medio-basse - un suggerimento che travalica $\mathrm{i}$ limiti del mondo inglese.

L'Italia del Settecento non è da meno di altri paesi dell'Europa occidentale nel contribuire alla riflessione e al progresso della scienza medica. Un confronto importante, non privo di ambigui punti di sovrapposizione, caratterizza il dibattito fra medicina 'razionale' e medicina 'empirica' che aveva al centro l'antica questione del valore dell'anatomia nell'arte del curare. Il dibattito aveva percorso tutta la storia sanitaria occidentale fin dal tempo dei greci. La novità era comunque rappresentata, nel Settecento, dalla prospettiva 'meccanicistica' nella spiegazione delle funzioni corporee fisiologiche e delle loro alterazioni patologiche. Massimo promotore in Italia di questo spirito interpretativo fu Marcello Malpighi che orientò e influenzò i medici 'meccanicisti' (definiti pure 'razionali' o 'dogmatici' sul solco dell'antica contesa ellenistica) raccolti anche sotto altre etichette terminologiche: iatromeccanici, iatrofisici, iatrochimici e così via, a secondo della particolare declinazione concettuale che li spingeva ad agire. A questi si opponevano gli 'empirici' secondo i quali la conoscenza umana era limitata nell'esplorazione delle 'cause remote' o 'interne' dei fenomeni e dunque doveva accontentarsi di sondarli soltanto sulla base di quanto poteva essere percepito attraverso l'osservazione. In realtà i due schieramenti non si contrapponevano sempre in maniera spiccata e potevano stabilire punti di contatto significativi. Fabio Zampieri si addentra lungo la selva delle discussioni accese che, a partire da Malpighi, si svilupparono ampiamente nel corso del XVIII secolo e ruotarono attorno all'opera capitale di Giovan Battista Morgagni. Il suo De sedibus et causis morborum per anatomen indagatis, del 1761, fin dal titolo indicava una prospettiva nuova alla medicina, quella che prefigurava un'indagine sulle 'sedi' e le 'cause' delle malattie, un'opzione già presente ma minoritaria nella tradizione precedente dominata dalla teoria degli umori. Nell'antichità non erano mancati i tentativi di ascrivere i processi morbosi a lesioni specifiche, come si può verificare nei grandi testi classici di Ippocrate, Galeno e Celso e, soprattutto, del medico greco Areteo di Cappadocia. Comunque, a partire da Morgagni, com'è noto, si fa risalire la grande fioritura dell'anatomia patologica con la descrizione delle alterazioni morfologiche negli organi corporei. Questa centralità nel tentativo di localizzare i processi morbosi accelerò la rottura 
del paradigma egemonico umorale risalente al mondo greco. In realtà, come mostra Zampieri, il testo di Morgagni non è un trattato di anatomia patologica, come la intendiamo oggi, ma un'opera di anatomia clinica. In altre parole, fu il risultato del tentativo sistematico di correlare i sintomi rilevati nei pazienti con specifiche lesioni d'organo scoperte durante la dissezione post mortem. In fondo, le recenti tecniche diagnostiche per immagini, RMN e TAC, realizzano in vita quanto nel De sedibus era possibile verificare soltanto con il decesso. Morgagni era un grande ammiratore di Malpighi e del suo spirito iatromeccanico, dunque anatomico, nell'esplorazione del corpo; tuttavia, la grande attenzione da lui riservata alle storie cliniche dei pazienti, centrale nella medicina empirica, lo pone in una specie di chiasma che congiunge questi due grandi filoni in una nuova sintesi unitaria destinata a un grande futuro.

Luca Borghi dedica le sue riflessioni a Giovanni Maria Lancisi, esponente importante della medicina a cavallo fra XVII e XVIII secolo. 'Caleidoscopica figura', come la definisce l'autore del saggio, medico di tre papi, questa complessa personalità fu studioso valente di anatomia, di clinica -il suo nome è tuttora ricordato per le 'strie di Lancisi' e per il 'segno di Lancisi' - e anche promotore della costruzione del primo teatro anatomico permanente a Roma nel 1688. Molto noto anche come indagatore della malaria (e della sua cura con le 'pillole di China'), fu grande divulgatore del sapere medico attraverso la fondazione e promozione della propria biblioteca concepita fin dall'inizio -scrive Borghi - non solo per i medici e per gli studenti di medicina ma per pubblica utilità. Questa istituzione diventò il fulcro degli incontri di studio per medici e chirurghi che andranno a regolarizzarsi nell'ambito dell'Accademia Lancisiana da lui fondata. Spirito indipendente, il medico romano tenne il 25 aprile 1715 la prolusione inaugurale del sodalizio davanti a una folla numerosa e a 13 cardinali, di fronte ai quali ebbe l'ardire di elogiare Galileo Galilei e Cartesio. Il saggio si sofferma sui molti aspetti dell'attività scientifica, accademica e didattica di Lancisi, che aveva contribuito a rendere la città dei papi un centro dove, conclude Borghi, «la ricerca scientifica e il sapere medico potevano dialogare alla pari con quelli delle nazioni europee più evolute».

Con il saggio di Gianni Iotti, ci si sposta dall'Italia alla Francia, e dai medici di professione agli uomini di cultura che, in quanto tali, sono irrimediabilmente attratti da questioni medico-scientifiche. Ne è chiaro esempio Denis Diderot che, negli ultimi anni della sua vita (dal 1769 al 1784), si dedicò principalmente a comporre i fondamentali Élements de 
physiologie: dedicati al naturalista Albrecht von Haller, possono essere considerati il testamento ideologico dell'enciclopedista francese. Iotti evidenzia come in questa opera il rifiuto di una teoria antropocentrica discenda dall'avversione dell'autore verso una superiorità e centralità ontologica dell'uomo nel contesto del sistema biologico, ma spiani tuttavia il cammino a sviluppi futuri nei campi dell'estetica, della letteratura e dell'espressione artistica, suggerendo una centralità dell'bomme sensible che trova riscontro nelle stesse opere letterarie e teatrali di Diderot, riformatore del teatro moderno. In questo contesto, va ricordato l'importante contributo dato dalla Scuola di Montpellier allo studio delle passioni in relazione all'empirismo sperimentale, che si era lasciato alle spalle la iatromeccanica: Théophile de Bordeu, capo di questa scuola, era grande amico di Diderot. Al centro dello schema vitalistico di Diderot era posta la molecola organica, che già Buffon e Maupertuis consideravano elemento primo e ultimo dell'intero organismo vivente. Questa si collegava alle fibre e alle membrane, i cui sviluppi erano concepiti in base a una temporalità specifica che, nella concezione di Diderot, ne accompagnava il processo di trasformazione. Tutto questo complesso sistema era volto a fondare una visione dell'uomo laica e materialista, e una medicina psicosomatica tesa tra la vita e la morte di ogni singolo elemento della struttura complessiva, che solo in uno stadio finale subiva il destino di una morte definitiva dell'intero organismo. Iotti precisa opportunamente che «il 'trasformismo' virtuale di Diderot si inscrive ancora nella visione linearista della 'catena degli esseri' di ascendenza idealistico-platonica che si ritrova anche in Linneo». Comunicare la scienza significa, per lo studioso francese, muoversi in uno scenario di un teatro dei nervi in cui l'immaginazione (precorrendo la scoperta dell'inconscio freudiano) si popola di immagini tra le quali è possibile vagabondare con rinnovata curiosità e con infantile entusiasmo.

Come Diderot uomini di cultura, anche se diversi nell'interpretare le istanze dei Lumi, sono i fratelli Verri e i loro collaboratori nell'impresa del Caffè, il cui interesse per le tematiche mediche viene esplorato da Gianmarco Gaspari. In sintonia con le istanze di rinnovamento propugnate dalla rivista, è chiara la presa di posizione a favore della medicina 'nuova': una medicina fondata su un approccio scientifico che supera, senza rinnegarlo, quello esperienziale, e che ha un ruolo importante nella vita sociale come in quella individuale. Dal punto di vista stilistico e comunicativo, poi, di medicina si parla nel Caffè mediante la 
formula, caratterizzante la rivista, dell'intrattenimento erudito. Nel proprio saggio, Gaspari mostra innanzitutto come gli estensori del Caffè alludano a tematiche mediche in vari articoli in cui altro è l'oggetto principale del discorso, perché in modi e contesti diversi le malattie possono essere combattute ed evitate $\mathrm{o}$, ancora meglio, può venire promosso il benessere della popolazione. Non mancano tuttavia articoli di diretta pertinenza medica, a partire da quello intitolato esplicitamente Medicina, secondo Gaspari non certo uno dei migliori di Pietro Verri. Sempre Pietro, tuttavia, è l'autore di quello che può senz'altro essere considerato il contributo d'argomento medico più importante della rivista, Sull'innesto del vaiuolo - essendo l'inoculazione, com'è noto, una pratica innovativa, molto controversa ai tempi, e non solo in Italia. Il saggio di Gaspari si espande poi a indagare altri interessanti aspetti del rapporto tra medicina e vita sociale, così come sono trattati nelle pagine del Caffè, inclusa la presentazione - come poteva mancare? - dei benefici effetti, sul corpo e sulla mente, dell'uso del caffè. Le sezioni conclusive dell'intervento di Gaspari rileggono l'impresa pubblicistica guidata da Pietro Verri, non solo alla luce dei modelli rappresentati dai giornali periodici inglesi, ma anche di un comune sentire - per quanto riguarda medici, medicine e malattie - che è un po' di tutta Europa.

Le comunicazioni presentate durante la giornata di studio all'Istituto Lombardo Accademia di Scienze e Lettere, e i saggi qui brevemente introdotti, non esauriscono certo il tema della riflessione settecentesca sulla medicina, ma ne suggeriscono le dimensioni, l'ampiezza e la profondità. $\mathrm{Ma}$, ancora di più e ancora più utilmente, richiamano quanto ha ispirato il progetto della giornata di studio: la dimensione transnazionale del pensiero medico-scientifico, l'intersecarsi di tale pensiero con diverse forme della riflessione culturale e dell'espressione artistica, e il crescente impatto delle pratiche di divulgazione in ambito scientifico. Elementi questi che il secolo del Lumi ci ha lasciato in eredità. 